\title{
Aproximación a la educación para la paz en la enseñanza universitaria: Algunas actividades prácticas*
}

\author{
María Martínez Lirola' \\ Universidad de Alicante (España) \\ Research Fellow, Department of Linguistics and Modern Languages, \\ University of South Africa (UNISA) (Sudáfrica)
}

Recibido: julio 28 de 2015 - Revisado: 20 de octubre de 2015 - Aceptado: 3 de noviembre de 2015

Referencia norma APA: Martínez-Lirola, M. (2015). Aproximación a la educación para la paz en la enseńanza universitaria: Algunas actividades prácticas. Revista Cientifica Guillermo de Ockham, 13(2), 35-43.

\section{Resumen}

Este artículo presenta una propuesta pedagógica basada en la educación para la paz en una clase de lengua inglesa en la educación universitaria, en concreto en el Grado en Estudios Ingleses. Se presentan algunas actividades cooperativas (análisis de textos escritos, escritura de un ensayo y participación en debates cooperativos) y se relacionan con algunos de los principios de la educación para la paz. Además, se señalará la pertinencia de las actividades propuestas para trabajar distintos tipos de competencias que sean de utilidad para el alumnado en el mercado laboral, como el respeto a la diversidad, la cooperación y la resolución pacífica de los conflictos. Se incluyen los resultados de una encuesta con el fin de conocer la opinión del alumnado sobre la educación para la paz y las competencias y destrezas que ha desarrollado. Una de las principales conclusiones es que las actividades propuestas contribuyen a potenciar las relaciones interpersonales pacíficas entre el alumnado; además, las actividades presentadas establecen relaciones entre lo que se enseña en el aula y la vida real.

Palabras clave: Educación para la paz, aprendizaje cooperativo, competencias, proceso de enseńanza-aprendizaje, universidad

\section{Approximation of education for peace in superior education: some practical activities}

\section{Abstract}

This paper offers a pedagogical proposal based on education for peace in an English classroom in University education, in the degree of English Studies. Some cooperative activities are presented (analysis of written texts, writing of an essay and participation about cooperative debates) and they are related with some of the principles of education for peace. Moreover, the relevance of the activities proposed to work with different types of competences that will be useful for students in the labour market such as respect for diversity, cooperation and the peaceful solving of conflicts will be pointed out. The results of a survey are included in order to know students' opinions on education for peace and the competences and skills they have developed. One of the main conclusions is that the proposed activities contribute

\footnotetext{
* Este artículo se deriva de la investigación de redes de investigación en docencia universitaria Experiencias de aprendizaje cooperativo y su relación con la multimodalidad en la adquisición de competencias, llevada a cabo desde noviembre de 2014 hasta noviembre de 2015.

1. Doctora en Filología Inglesa y profesora titular de la Universidad de Alicante. Directora del grupo de investigación Análisis Crítico del Discurso Multimodal. Research Fellow, Department of Linguistics and Modern Languages, University of South Africa (UNISA). Dirección postal: Universidad de Alicante, Departamento de Filología Inglesa, Ap. 99 E-03080 Alicante (Espańa).E-mail: maria.lirola@ua.es
} 
to strengthen peaceful interpersonal relationships between students. Additionally, the activities described in the article establish relationships between what is taught in the classroom and in real life.

Keywords: Education for peace, cooperative learning, competences, teaching-learning process, university.

\section{Abordagem à educação para a paz no ensino superior: algumas atividades práticas}

\section{Resumo}

Este artigo apresenta uma abordagem pedagógica baseada na educação para a paz em uma classe de Inglês no ensino superior, em particular na Licenciatura em Estudos Ingleses. Algumas actividades de cooperaçáo (análise de textos, escrita e participaçáo em discussóes cooperativas ensaio) são apresentadas e relacionadas com alguns dos princípios da educaçáo para a paz. Além disso, a relevância das propostas para trabalhar diferentes tipos de habilidades que são úteis para os estudantes no mercado de trabalho e respeito à diversidade, a cooperação e as actividades de resolução pacífica de conflitos indicam. Os resultados de uma pesquisa para conhecer a opinião dos alunos sobre educação para a paz competências e habilidades desenvolvidas estão incluídos. Uma das principais conclusóes é que as actividades propostas contribuem para promover relaçôes pacíficas entre os alunos. Além disso, as atividades apresentadas estabelecer relaçôes entre o que é ensinado na sala de aula e na vida real.

Palavras-chave: Educação para a paz, a aprendizagem cooperativa, habilidades de ensino-aprendizagem, universidade

\section{Introducción}

La universidad del siglo XXI se caracteriza por el empleo de metodologías activas con el fin de que el alumnado sea activo en todo el momento del proceso de enseńanzaaprendizaje tal y como requiere el Espacio Europeo de Educación Superior (EEES) (López-Noguero, 2005; Rué, 2007). Esto implica que lo que se enseña y se aprende en las aulas universitarias ha de tener relación directa con lo que demandan las sociedades actuales, y en concreto el mercado laboral. En este sentido, es importante que la universidad no se limite solo a enseñar conocimientos, sino también competencias que contribuyan a la formación integral del alumnado y le ayuden a configurar una ciudadanía activa con el fin de adaptarse con facilidad a los cambios constantes de la sociedad.

En estos tiempos de crisis económica se observan ciertas actitudes de desánimo en todas las capas de la sociedad, lo cual se traduce en actitudes violentas como expresión de frustración interna y desesperanza. Por esta razón, nos parece importante trazar una propuesta educativa en la enseńanza superior enmarcada en los principios de la educación para la paz, con el fin de que los educandos se apropien de competencias y desarrollen actividades a la luz de estos principios. De este modo, podrán aplicar lo que aprenden al mercado laboral, por lo que estaremos formando profesionales activos y comprometidos capaces de ofrecer respuestas pacíficas y resolver los conflictos de forma no violenta.

Los principales objetivos de este artículo consisten en diseñar una propuesta de actividades enmarcadas en los principios de la educación para la paz, con el fin de que el alumnado conozca los principios de la educación para la paz por medio de la temática de las distintas actividades. Además, nos proponemos observar si con estas tareas esta colectividad adquiere competencias sociales. Para ello se diseñó una encuesta cuyos resultados se presentan en la discusión.

Optar por un enfoque basado en la educación para la paz, ofrece la oportunidad de emplear la pedagogía para producir transformaciones sociales, en virtud de que favorece la enseñanza de valores en el proceso de enseñanzaaprendizaje (Harris \& Synott 2002). Además, la educación para la paz ofrece propuestas para la prevención de los conflictos o para su resolución de forma pacífica, tanto en el entorno educativo como en la sociedad (Baesler \& Lauricella, 2014; Galtung \& Jacobsen, 2000; Lum, 2013; Muñoz, 2004; Oyesola, 2005; Page, 2004; Salomon, 2002; Sandy, 2001). De ahí que sea un enfoque adecuado para trabajar en las aulas de cualquier nivel educativo con el fin de mejorar el proceso de enseńanza-aprendizaje y contribuir a la construcción de un mundo mejor. 
La educación para la paz potencia la formación del alumnado en la adquisición de competencias sociales, como la resolución de conflictos, la capacidad crítica, la toma de decisiones y la cooperación, entre otras. En este sentido, es evidente que el estudiante ha de ser activo y no pasivo (Korostelina, 2012; Martínez-Lirola, 2013) cuando aprende, de modo que se convierta en agente de los cambios que la sociedad necesita para mejorar (Bajaj, 2008; Galtung \& Udayakumar, 2013; Harris \& Morrison, 2003; Page, 2008). Es de esta manera que se entiende la educación para la paz vinculada a la educación para los derechos humanos.

\section{Aproximación a la educación para la paz}

Los orígenes de la educación para la paz se remontan a 1945 cuando se crean las Naciones Unidas con el fin de proteger las futuras generaciones de la guerra, preservar la dignidad de los seres humanos y asegurar la igualdad entre mujeres y hombres, entre otros. La educación para la paz surge como uno de los medios para conseguir estos objetivos, comoquiera que su objetivo es el pleno desarrollo de la personalidad humana y el fortalecimiento del respeto a los derechos humanos y las libertades fundamentales. Promueve "la comprensión, la tolerancia y la amistad entre todas las naciones, grupos étnicos o religiosos" y "las actividades de las Naciones Unidas para el mantenimiento de la paz" (Declaración Universal de los Derechos Humanos, 1948: art. 26).

La educación para la paz hace referencia al proceso de favorecer las destrezas, los conocimientos, las actitudes y los valores necesarios con el fin que las personas opten por prevenir el conflicto y la violencia, resolver el conflicto de manera pacífica y crear unas condiciones que permitan la presencia de la paz tanto a nivel intrapersonal, interpersonal nacional e internacional. Harris y Synott (2002) proponen la siguiente definición de educación para la paz: "Por educación para la paz, nos referimos a la enseńanza de los encuentros que atraen a la gente hacia fuera de sus deseos de paz y les proporcionan alternativas no violentas para la gestión de conflictos, así como las habilidades para el análisis crítico de los arreglos estructurales que legítima y producen la injusticia y la desigualdad" (p. 4).

Son diversas las perspectivas desde las que se puede entender la paz en general y la cultura de paz en particular: paz como ausencia de guerra; paz como un equilibrio de fuerzas en el sistema internacional; paz como negativa (no guerra) y como positiva (no violencia estructural); paz feminista: macro y micro niveles de la paz; paz holística con el medioambiente, y paz holística interna y externa (Groff \& Smoker, 1996).
Este artículo se centra en la tercera perspectiva, la cual presta atención a la evolución de paz negativa a paz positiva, con el fin de hacer referencia a la ausencia de violencia estructural. De este modo, la paz no se entiende solo como ausencia de guerra, sino también relacionada con otras cuestiones sociales, económicas y políticas, entre las que se encuentra la educación.

Los objetivos fundamentales de la educación para la paz incluyen, en primer lugar, comprender las manifestaciones de la violencia y el desarrollo de capacidades para responder a la violencia de forma pacífica (Galtung, 1975; 1988; 1996; 2004; Galtung \& Jacobsen, 2000). En segundo lugar, ofrece soluciones para la resolución pacífica de los conflictos. En tercer lugar, potencia el desarrollo de destrezas como la comunicación y la cooperación por su importancia en la resolución de conflictos. En este sentido, es fundamental el respeto por uno mismo y por los otros (Kester, 2012; 2013).

Son múltiples los trabajos de los últimos años en el marco de la educación para la paz. La mayoría de ellos se centra en la importancia de la resolución pacífica de los conflictos, por ser este uno de los objetivos fundamentales de la educación para la paz (Bar-Tal, 2002; Danesh \& Danesh, 2002a; 2002b; 2004; Davies, 2005; Isumonah, 2005; Johnson, Johnson \& Tjosvold, 2000; Sandy, 2001). Estos estudios coinciden en que la negociación es la principal respuesta de la educación para la paz y para la resolución de conflictos:

Las diversas definiciones y conceptos [...] tienen en común la idea que la educación para la paz es para negociar la violencia y el conflicto y que además sirve para promover una cultura de paz y contrarrestar la cultura de la guerra. Subyacente a este denominador común es la suposición que la educación de la paz, pese a servir a otros objetivos como los derechos humanos y la democracia, es ante todo un proceso educativo que opera dentro del contexto de la guerra, la amenaza, la violencia y los conflictos que se ocupa de las actitudes, creencias, atribuciones, competencias y comportamientos (Salomon \& Cairns, 2010, p. 5).

Otros estudios se centran en describir los principios de la educación para la paz y las ventajas de su aplicación en los distintos niveles educativos (Bajaj \& Chiu, 2009; Danesh, 2008; Harris \& Morrison, 2003; Page, 2004; 2008; Salomon \& Nevo, 2002; Tal-Or, Boninger\& Gleicher, 2002). Otras investigaciones describen experiencias prácticas con las que se potencia una enseńanza de calidad enmarcada en los parámetros de la educación para la paz (Clarke-Habibi, 2005; Hicks, 2004; Kester \& Booth, 2010; Salomon, 2002; Timpson, 2002). 
La educación para la paz supone llevar a la práctica una pedagogía que contribuya al refuerzo de la armonía en el mundo por medio del diálogo, el respeto a la diversidad y la resolución pacífica de los conflictos que surgen en el aula y en el día a día. Además, la educación para la paz lleva consigo un respeto por el entorno y por todas las formas de vida, de ahí que se considere un enfoque adecuado para promover la integración en el aula y ver las diferencias entre los distintos seres humanos como oportunidades para enriquecerse.

En la actualidad se desarrolla un movimiento global de personas que reconocen que la educación para la paz desempeña un papel fundamental en la transformación social. El número de docentes que en diferentes lugares del mundo asume el reto de la educación para la paz aumentado día a día en los últimos ańos. Ello implica la puesta en práctica de actividades que promuevan la paz, rechacen la injusticia y prevengan los conflictos violentos en las aulas. La educación para la paz ofrece recursos y potencialidades para dar más relevancia a un modelo de educación en el siglo XXI basado en el respeto por el otro, pues ofrece respuestas a muchas de las problemáticas de la sociedad actual donde predominan los conflictos, el individualismo y las actitudes violentas.

\section{Participantes, contexto y metodología}

La propuesta de actividades que se presentan en este artículo se llevó a cabo en el grado en Estudios Ingleses de la Universidad de Alicante, en concreto en la asignatura obligatoria Lengua Inglesa $\mathrm{V}$, de tercer curso. El fin principal de esta asignatura consiste en que los alumnos matriculados mejoren su nivel de escucha, habla, lectura, escritura e interacción con el fin de poder alcanzar un nivel $\mathrm{C} 1$ en inglés, una vez que cursen las dos asignaturas de lengua que siguen a esta (Lengua Inglesa VI y VII) en el segundo cuatrimestre de tercero y en el primer cuatrimestre de cuarto.

La asignatura está diseñada para trabajar durante ciento cincuenta horas: noventa fuera del aula con el fin de que el alumnado trabaje de manera grupal e individual en las actividades propuestas, y sesenta teórico-prácticas en el aula. En el curso académico 2014-2015 hubo ciento veinticinco personas matriculadas, de las cuales trece obtuvieron una beca Erasmus y estudiaron una asignatura similar en otras universidades europeas.

Respecto a la metodología de la asignatura, se optó por una disciplina interactiva que promoviera la participación activa del alumnado durante el proceso de enseñanza- aprendizaje. Debido al gran número de personas matriculadas, la clase se organizó en grupos de cinco o seis personas para llevar a cabo las actividades cooperativas propuestas en el apartado que sigue.

La asignatura se imparte durante cuatro horas de clase semanales: una teórica y tres prácticas. En la hora teórica, se ofrece una introducción a los principales aspectos de la escritura académica con especial atención hacia las características fundamentales de los diferentes tipos de textos y sus relaciones con el contexto en que se emplean. Además, se analizan los recursos cohesivos que aparecen en los textos y se presenta la estructura del ensayo académico, entre otras cuestiones. La segunda hora se dedica al desarrollo de la destreza oral, para lo cual un grupo compuesto por unas cinco o seis personas expone en clase una presentación oral grupal sobre un tema social de actualidad. La tercera hora es utilizada para avanzar en cuestiones de gramática del nivel C1, como el correcto uso de los adverbios y los artículos, la voz pasiva, el estilo indirecto, etc. Tras una breve explicación teórica, se corrigen los ejercicios gramaticales con el fin de que el alumnado lleve a la práctica lo aprendido. La cuarta hora consiste en la participación en un debate cooperativo preparado por el grupo que ha hecho la presentación oral esa semana. El tema del debate debe ser el mismo que el de la presentación oral, con el fin de que el alumnado practique el vocabulario aprendido, involucre a toda la clase en el tema objeto de estudio y trabajeel pensamiento crítico.

La planificación de las clases descrita en el párrafo anterior implica que la asignatura está centrada en el alumnado en lugar del profesorado. Es decir, los estudiantes son los protagonistas del proceso de enseñanza-aprendizaje y por lo tanto desempeñan un papel fundamental a la hora de tomar decisiones en la clase, hecho que contribuye a la creación de un clima pacífico, dinámico y efectivo de aprendizaje de la lengua extranjera.

En las secciones que siguen se presenta una propuesta de actividades enmarcadas en un plan de educación para la paz y se exponen los resultados de una encuesta dirigida a conocer la opinión del alumnado sobre la educación para la paz y las competencias y destrezas que ha desarrollado.

\section{Propuesta de actividades que potencian la educación para la paz}

\section{Análisis de textos escritos y escritura de un ensayo}

Al tratarse Lengua Inglesa $\mathrm{V}$ de una asignatura en la que el alumnado matriculado tiene un nivel avanzado de 
inglés, nos pareció importante seleccionar temas globales desde el principio del cuatrimestre con el fin de trabajar tanto las destrezas orales como las escritas. Con respecto a la escritura, el objetivo es seleccionar distintos tipos de texto con miras a que el alumnado perciba sus principales características formales y estructurales, así como su función, con el objeto de que escriba textos adecuados a un contexto determinado, efectivos desde el punto de vista lingüístico y estructural y con un vocabulario específico. Al principio del cuatrimestre, el docente explicó los principales recursos cohesivos en inglés (referencia, sustitución, elipsis, conjunción y cohesión léxica) con el fin de que los estudiantes fueran conscientes de los recursos que ofrece la lengua inglesa para dotar a un texto de cohesión. Estos recursos fueron ilustrados en textos sobre ámbitos globales como los siguientes: inmigración, situación de las mujeres en el mundo, cambio climático, salud, diferencias culturales, racismo y la paz, entre otros. Trabajar estos campos entraña la adquisición de un vocabulario relacionado con determinadas áreas semánticas relacionadas con asuntos sociales y una cultura de paz, todo lo cual contribuye al desarrollo de la educación para la paz. Estos estudios ofrecieron al estudiante la oportunidad de desarrollar el pensamiento crítico, aproximarse y entender a otras personas culturalmente diferentes y sentirse ciudadano activo en una sociedad globalizada, en la que las diferencias individuales y culturales han de ser tenidas en consideración. Un ejemplo claro de esto es el análisis en profundidad de uno de los discursos más conocidos del expresidente sudafricano Nelson Mandela, en concreto, el que pronunció en Pretoria el 10 de mayo de 1994 cuando fue proclamado presidente. El alumnado analizó los argumentos centrales y los principales recursos cohesivos empleados por Mandela para expresar los significados y destacar alguna información como importante, de acuerdo con lo prescrito por el docente.

Otra actividad empleada para el análisis de los principales recursos cohesivos en inglés, consistió en dividir el discurso de Martin Luther King I have a dream en ocho apartados y asignarles a sendos grupos establecidos en clase unos párrafos, con el fin de que todo el curso analizara el discurso de forma cooperativa. De este modo, los educandos no solo trabajan un texto de corte social en el que se ponen de manifiesto las diferencias entre la población blanca y la población negra en la época de Luther King, sino que además participan activamente en una metodología cooperativa, toda vez que el resultado final de la actividad depende del análisis efectuado por cada grupo. De esta forma, en lugar de promover un espíritu competitivo, se fomenta la cooperación, idea que encaja con los principios de la educación para la paz a los que nos referiremos en la discusión.

Una vez que se ha practicado el análisis de textos reales, se pidió al alumnado la composición de un ensayo en el que se establecieran diferencias y semejanzas entre Martin Luther King y Mandela, ello con el fin de que los educandos trabajaran el pensamiento crítico. Además, se les pidió que expresaran su opinión sobre la existencia de este tipo de líderes en la actualidad y su repercusión en los avances de la humanidad.

\section{Debates cooperativos}

Para el desarrollo de esta área, el alumnado se dividió en grupos de cinco personas, con el fin de preparar una presentación oral cooperativa y un debate. En la primera clase, el profesor expuso los principales aspectos relacionados con una presentación oral efectiva y las partes y recursos que debían emplearse en su preparación. En las tres primeras clases prácticas, el docente preparó los siguientes debates relacionados con aspectos de la educación para la paz, con el fin de que el alumnado aprendiera técnicas que aplicarían en el debate.

La primera actividad consistió en dividir la clase en seis grupos y numerar a las personas del uno al seis, a fin de que se trabajara con personas distintas. Un grupo representaba un conjunto de alumnos de distintos niveles educativos que sufría discriminación en clase en razón de su sexo y raza y buscaba una solución. El resto de los grupos representaban distintos colectivos y debían ofrecer soluciones a partir del papel que se les había asignado: personalidades políticas, representantes de las Naciones Unidas, rectores de universidad, directores de institutos y directores de colegio. Cada grupo debía nombrar un portavoz que expondría los problemas que sufría el grupo que representaba al alumnado y las soluciones en el caso del resto de los grupos. Al final, debía elegir al grupo que mejores soluciones había ofrecido, de modo que se trabajaba el pensamiento crítico, el liderazgo, la escucha activa y la solidaridad, entre otras competencias. Con esta actividad se invita al alumnado a buscar soluciones creativas contra la discriminación y a pensar en sus causas y consecuencias.

La segunda actividad consistió en trabajar sobre los derechos humanos. El maestro comenzó la clase preguntando al alumnado qué sabía al respecto y la respuesta fue prácticamente nada. A continuación, se presentó un video sobre la historia de los derechos humanos con el fin de sembrar algunos conocimientos previos y de 
nuevo se dividió la clase en seis grupos, pero en esta ocasión fue el alumnado el que los organizó con el fin de trabajar competencias relacionadas con la toma de decisiones y la organización. Cada grupo recibió el texto de la Declaración Universal de los Derechos Humanos completo, sin embargo, con el fin de que en la hora de clase se pudiera profundizar en todos los derechos, a cada grupo se le pidió que se centrara en los cinco indicados por el docente, con el fin de que reflexionara sobre ellos y pensara en situaciones en los que estos derechos no se respetan y en las posibles soluciones para su reivindicación y su visibilización. La mayoría de los grupos apuntan que la educación es la herramienta fundamental para que las personas reflexionen sobre la importancia de respetar los derechos humanos y señalan la dificultad para que se cumplan, especialmente en los países en los que no hay democracia. También ponen de manifiesto que el sistema capitalista no potencia los derechos humanos, pues lo único que importa es el capital y lo que las personas producen y consumen.

La tercera actividad -también relacionada con la educación para la paz- consistió en dividir la clase en seis grupos distintos a los de las sesiones anteriores con el fin de que el alumnado conociera toda la clase y se creara un buen clima de grupo. A dos grupos se les pidió que discutieran el tipo de enseñanza que tendrían basada en la educación para la paz; a otros dos una basada en la enseñanza tradicional centrada en el profesorado como centro del proceso de enseñanza-aprendizaje y al resto una basada en un profesorado que no tiene interés en enseñar y cuyo único interés es tener su sueldo a final de mes. La profesora nombró un líder en cada grupo con el fin de que las personas más tímidas participaran y asumieran protagonismo en el aula. Todos los grupos explicaron los principios del modelo de enseńanza que presentaban exponiendo aspectos relacionados con quién soy, qué pienso, qué siento y qué reivindico. A continuación, todos los miembros del grupo, excepto el líder, podían cambiar de equipo si el modelo presentado por otro grupo les parecía más efectivo para llevar a cabo un proceso de enseñanza-aprendizaje eficaz. Fue interesante observar cómo la mayoría del alumnado eligió el modelo basado en la educación para la paz por uno de los grupos, hecho que el docente empleó para señalar la importancia de la persuasión, pues aunque dos grupos tenían el mismo modelo de enseńanza, los argumentos eran diferentes.

\section{Discusión}

Las actividades presentadas en la sección anterior son un ejemplo de cómo se puede diseñar una asignatura de lengua extranjera sobre los principios de la educación para la paz. En primer lugar, se potencia que el alumnado sea activo durante todo el proceso de aprendizaje. Tanto las actividades relacionadas con el análisis de textos como con la participación en debates cooperativos, fomentan la interacción y la expresión de las opiniones personales de modo que se trabaja el pensamiento crítico. Esto implica la formación del alumnado como ciudadanía crítica y comprometida, al tomar conciencia de que su discurso y sus acciones tienen una repercusión social. Además, las actividades permiten el desarrollo de destrezas relacionadas con la escritura académica y hablar en público; es decir, se observa la utilidad de lo que se aprende para el futuro profesional del alumnado.

Para que el alumnado interactúe con libertad en clase es necesario construir un buen clima en el aula, de modo que no se tenga temor a intervenir por la ansiedad que produciría el cometer errores. Por esta razón, es necesario que el profesorado esté formado no solo en su materia sino también en didáctica y en competencias emocionales, con el fin de pueda gestionar adecuadamente el clima del aula y las emociones que se generan al poner en práctica una pedagogía activa como la que se propone en este artículo (Simon \& Pleschová, 2013). En este sentido, el profesor dejó claro al principio del curso académico que los errores no han de verse como algo negativo, sino como una oportunidad para aprender y mejorar. De este modo, el alumnado tiene más seguridad para participar al ver el error con otra perspectiva.

En segundo lugar, las actividades propuestas contribuyen a la formación integral del alumnado universitario, al llevar conllevar la reflexión activa sobre problemas sociales que permiten poner de manifiesto la formación en competencias, como el respeto a la diversidad de opiniones, la escucha activa y la cooperación, entre otras. Esto se puede ver claramente en los distintos debates descritos, en los cuales el alumnado tiene que interactuar representando distintos papeles y dialogar sobre asuntos fundamentales en las sociedades democráticas, como los derechos humanos o la educación. De esta forma, la asignatura introduce contenidos relacionados con la paz en el currículum a través de los temas de los textos seleccionados para la lectura y la escritura y los temas sociales que se han de tratar en clase para desarrollar las destrezas orales.

Un ejemplo claro de esto es el análisis del Discourse of Inauguration as President de Nelson Mandela (Asmal et al., 2004), pues en él se ofrece un planteamiento sociopolítico pacifista en la realidad social de Sudáfrica tras el apartheid, cuya característica fundamental era la segregación racial 
entre blancos y negros. En este sentido, trabajar con un texto de Mandela es también un ejemplo de la ideología pacifista de uno de los grandes líderes del siglo XX, pues a pesar de haber estado encarcelado durante años por su ideología, su respuesta fue siempre pacífica al apostar por la reconciliación, la justicia y la paz.

Lo mismo ocurre con el análisis de I have a dream. El hecho de que el alumnado analice con detalle los distintos recursos cohesivos empleados por Martin Luther King para transmitir su ideología pacifista y poner de manifiesto la necesidad de acabar con la desigualdad racial, envuelve una reflexión sobre la importancia de la igualdad y sobre las distintas formas de discriminación presentes en el siglo XXI y la necesidad de combatirlas desde una postura pacífica.

En tercer lugar, hemos de señalar que las metodologías activas conllevan en ocasiones conflictos entre los distintos integrantes de los grupos, por razones varias relacionadas con el reparto de tareas, la toma de decisiones y el liderazgo, entre otras. En este sentido, las actividades descritas permiten trabajar el principio de la educación para la paz relacionado con la resolución pacífica de conflictos, pues en todo momento el profesor puso de manifiesto que las diferencias en los grupos se resolverían por medio del diálogo y que si fuera necesario actuaría como mediador. La negociación ocupa un lugar importante en la organización de las actividades de los grupos y en la resolución pacífica de los conflictos. El docente insistió en que ver los conflictos como oportunidades para crecer ofrece la posibilidad de llevar a la práctica los principios de la educación para la paz, concretados mediante el desarrollo de las distintas actividades propuestas.

Las actividades descritas se pueden materializar en otros niveles educativos, pero el hecho de trabajar en la educación superior hace posible que los principios de la educación para la paz que se trabajan en cada actividad se expliciten, de modo que el alumnado sea consciente en todo el proceso de aprendizaje de lo que cada actividad lleva implícito. En este sentido, es importante señalar que estas labores contribuyen a la adquisición de competencias sociales, como la comunicación, la cooperación y la resolución de conflictos, de modo que se trabajan con la formación integral del alumnado y no solo con competencias que comporten la adquisición de conceptos.

Además, las actividades se han diseñado para que los educandos sean los protagonistas y el profesorado asuma un papel de guía, facilitador o tutor. Las actividades descritas implican que el alumnado "aprende haciendo", de modo que pone en práctica lo que aprende y establece relaciones entre lo que ocurre en las aulas y en la vida real.

Tal y como se ha señalado, además de diseñar unas actividades enmarcadas en una propuesta educativa integrada en la educación para la paz, también se pretendía conocer la opinión del alumnado sobre la educación para la paz y las competencias y destrezas que había desarrollado. Por esta razón, se preparó una encuesta cuyos resultados presentamos a continuación.

Con respecto a la primera pregunta, para casi totalidad del alumnado encuestado la paz es algo importante en su vida: $99,1 \%$ frente a un $0,9 \%$, obtenido de la respuesta que ofrecen dos personas. Llama la atención que al responder la segunda pregunta, muchos alumnos ponen de manifiesto que no conocen el enfoque de la educación para la paz, pues noventa y una personas frente a ciento cuarenta ofrecen una respuesta negativa a esta pregunta, lo que constituye un $39,4 \%$ frente a un $60,6 \%$ que afirma conocerlo.

Los resultados a la pregunta tercera son claros, pues casi la totalidad del alumnado encuestado $(97,4 \%)$ considera que el enfoque de la educación para la paz les puede ayudar en su vida diaria. Las razones para ofrecer una respuesta positiva varían; sin embargo, muchos señalan que este enfoque facilita la comprensión de distintos puntos de vista, la resolución pacífica de conflictos y por lo tanto la convivencia.

En relación con la pregunta cuarta, es interesante observar cómo el alumnado es consciente de las distintas competencias que ha adquirido con esta propuesta educativa, al destacarla cooperación $(75,8 \%)$, la comunicación $(68 \%)$, el respeto a la diversidad $(62,8 \%)$ y la resolución de conflictos $(45,8 \%)$.

Al preguntarles en la pregunta quinta cuáles destrezas han trabajado mayormente, destacan la destreza oral $(61,9 \%)$ y la interacción $(68,4 \%)$, de modo que queda claro que las actividades y el enfoque elegido ayudan al alumnado a mejorar su expresión oral en lengua inglesa, aspecto fundamental para su incorporación al mercado laboral.

\section{Conclusiones}

Este artículo presenta un ejemplo práctico de cómo una asignatura de lengua inglesa puede transformarse, de modo que además de aprender las distintas destrezas que se necesitan desarrollar en una lengua extranjera (lectura, escritura, habla, escucha e interacción) se incorporen algu- 
nos principios básicos de la educación para la paz, como la resolución pacífica de conflictos, el desarrollo de valores y el respeto a la diversidad, entre otros. De este modo, las prácticas docentes contribuyen a la construcción de un mundo mejor al apostar por la dimensión social de la paz.

Las actividades presentadas en este artículo pretenden ilustrar una propuesta pedagógica activa que potencie las relaciones interpersonales pacíficas entre el alumnado. En este sentido, es determinante seleccionar actividades cooperativas que fomentan la interacción durante el proceso de enseńanza-aprendizaje y permitan al alumnado discutir, negociar, tomar decisiones o repartir las tareas de forma equitativa. De esta forma, tanto las actividades propuestas como la pedagogía empleada contribuyen a transformar la sociedad al desarrollar competencias valoradas en el mercado laboral, como la cooperación, la escucha activa, el reparto de tareas o la resolución pacífica de los conflictos.

En consecuencia, con las actividades presentadas en este artículo se establece una relación entre el trabajo que se hace en clase y la vida real, pues las actividades que se efectúan van encaminadas no solo a que el alumnado mejore su nivel de inglés, sino también a que se convierta en ciudadanía activa y crítica de modo que se sienta protagonista en la construcción de la sociedad del siglo XXI.

\section{Referencias}

Bajaj, M., \& Chiu, B. (2009). Education for Sustainable Development as Peace Education. Peace and Change, 34(4), 441-455.

Baesler, J. E., \& Lauricella, S. (2014). Teach peace!: Assessing instruction of the nonviolent communication and peace course. Journal of Peace Education, 11, 1, 46-63.

Bar-Tal, D. (2002). The elusive nature of peace education. En G. Salomon \& B. Nevo (Eds.), Peace education: The concepts, principles, and practices around the world (pp. 27-36). Mahwah, NJ: Lawrence Erlbaum Associates.

Clarke-Habibi, S. (2005). Transforming Worldviews: The Case of Education for Peace in Bosnia and Herzegovina. Journal of Transformative Education, 3(1), 33-56.

Danesh, H. B. (2008). The education for peace integrative curriculum: Contents, concepts, and efficacy. Journal of Peace Education, 5(2), 157-174.

Danesh H. B., \& Danesh, R. P. (2002a). Has conflict resolution grown up?: toward a new model of decision making and conflict resolution. International Journal of Peace Studies, $7(1), 59-76$.
Danesh H. B., \& Danesh, R. P. (2002b). A consultative conflict resolution model: beyond alternative dispute-resolution. International Journal of Peace Studies, 7(2), 17-33.

Danesh, H., B., \& Danesh, R. (2004). Conflict-free conflict resolution (CFCR): process and methodology. Peace and Conflict Studies, 11(2), 55-84.

Davies, L. (2005). The edge of chaos: explorations in education and conflict. En J. Zajda (Ed.), International Handbook on Globalisation, Education and Policy Research (pp. 631-642). Springer: The Nederlands.

Declaración Universal de los Derechos Humanos. (1948). Resolución de la Asamblea General 217A (III) del 10 de diciembre 10, 1948; U.N. Doc A/810, 71 (1948), Artículo 26.

Galtung, J. (1975). Peace: Research, education, action. Essays in Peace Research. Copenhagen: Christian Ejlers.

Galtung, J. (1988). Peace and social structure. Essays in Peace Research. Copenhagen: Christian Eljers.

Galtung, J. (1996). Peace by peaceful means: Peace and conflict, development and civilization. Londres: Sage.

Galtung, J. (2004). Trascend and Transform an Introduction to Conflict Work. London: Pluto Press.

Galtung, J., \& Jacobsen, C. G. (2000). Searching for Peace: the Road to Transcend. London: Pluto Press.

Groff, L., \& Smoker, P. (1996). Creating global/local cultures of peace. En Unesco, From a culture of violence to a culture of peace (pp. 103-128). Paris: Unesco.

Harris, I., \& Morrison, M. (2003). Peace Education. Jefferson, NC: McFarland \& Co.

Harris, I., \& Synott, J. (2002). Peace education for a new century. Social Alternatives, 21(1), 3-6.

Hicks, D. (2004). Teaching for tomorrow: How can future studies contribute to peace education? Journal of Peace Education, 1(2), 165-178.

Isumonah, V. A. (2005). Problems of peacemaking and peacekeeping. En Albert, I. O. (Ed.), Perspectives on Peace and Conflict In Africa (pp. 198-216). Ibadan: Peace and Conflict Studies Programme.

Johnson, D., Johnson, R., \& Tjosvold, D. (2000). Constructive controversy: the value of intellectual opposition. In M. Deutsch \& P. T. Coleman (Eds.), The Handbook of Conflict Resolution: Theory and Practice (pp. 65-85). San Francisco, CA, Jossey-Bass.

Kester, K. (2012). Peace Education Primer. Journal of Global Citizenship \& Equity Education, 2(2), 62-75.

Kester, K. (2013). Peace education: an impact assessment of a case study of UNESCO-APCEIU and the university for peace. Journal of Peace Education, 10(2), 157-171.

$42<$ Universidad de San Buenaventura, Cali - Colombia 
Kester, K., \& Booth, A. (2010). Education, peace and Freire: A dialogue. Development, 53(4), 498-503.

Korostelina, K. V. (2012). Introduction. En K. V. Korostelina (Ed.), Forming a Culture of Peace. In Reframing Narratives of Intergroup Relations, Equity, and Justice (pp. 1-14). NY: Palgrave Macmillan.

López-Noguero, F. (2005). Metodología participativa en la enseñanza universitaria. Madrid: Narcea.

Lum, J. (2013). Peace education: past, present, and future. Journal of Peace Education, 10(3), 215-229.

Asmal, K., Chidester, D., \& James. W. (2004). Nelson Mandela. In His Own Words. From Freedom to the Future. Lancaster: Abacus.

Martínez-Lirola, M. (2013). Ejemplos de la relación entre el aprendizaje cooperativo y la adquisición de competencias interpersonales en una clase de lengua inglesa. Encuentro Revista de investigación e innovación en la clase de idiomas, 22, 73-83.

Muñoz, F. (2004). Manual de Paz y Conflictos. Granada: Universidad Granada.

Oyesola, D. (2005). Environmental Degradation and Peace Studies. In I. O. Albert (Ed.), Perspectives on Peace and Conflict in Africa (pp. 253-267). Ibadan: Peace and Conflict Studies Programme.

Page, J. S. (2004). Peace Education: Exploring Some Philosophical Foundations. International Review of Education, 50(1), 3-15.

Page, J. S. (2008). Peace Education: Exploring Ethical and Philosophical Foundations. Charlotte: Information Age Publishing.
Rué, J. (2007). Enseñar en la Universidad. El EEES como reto para la Educación Superior. Madrid: Narcea.

Salomon, G. (2002). The nature of peace education: Not all programs are created equal. In G. Salomon \& B. Nevo (Eds.), Peace education: The concepts, principles, and practices around the world (pp. 3-14). Mahwah, NJ: Lawrence Erlbaum Associates.

Salomon, G., \& Cairns, E. (2010). Handboook on Peace Education. NY: Taylor \& Francis.

Salomon, G., \& Cairns, E. (2010). Peace education. Setting the scene. In G. Salomon \& E. Cairns (Eds.), Handbook on Peace Education (pp. 1-7). NY: Taylor and \& Francis.

Salomon, G., \& Nevo, B. (2002). Peace Education: The Concept, Principles and Practices around the World. Mahwah, NJ: Lawrence Erlbaum.

Sandy, S. (2001). Conflict resolution in schools: "Getting there". Conflict Resolution Quarterly, 19(2), 237-250.

Simon, E., \& Pleschová, G. (2013). Teacher Development in Higher Education. Existing Programs, Program Impact, and Future Trends. Londres/Nueva York: Routledge.

Tal-Or, N., Boninger, D. \& Gleicher, F. (2002). Understanding the Conditions and Processes Necessary for Intergroup Contact to Reduce Prejudice. In G. Salomon \& B. Nevo (Eds.), Peace Education, The Concept, Principles, and Practices Around the World. (pp. 89- 108). Mahwah, NJ: Lawrence Erlbaum Associates Publishers.

Timpson, W. (2002). Teaching and Learning Peace. Madison, WI: Atwood Publishing. 